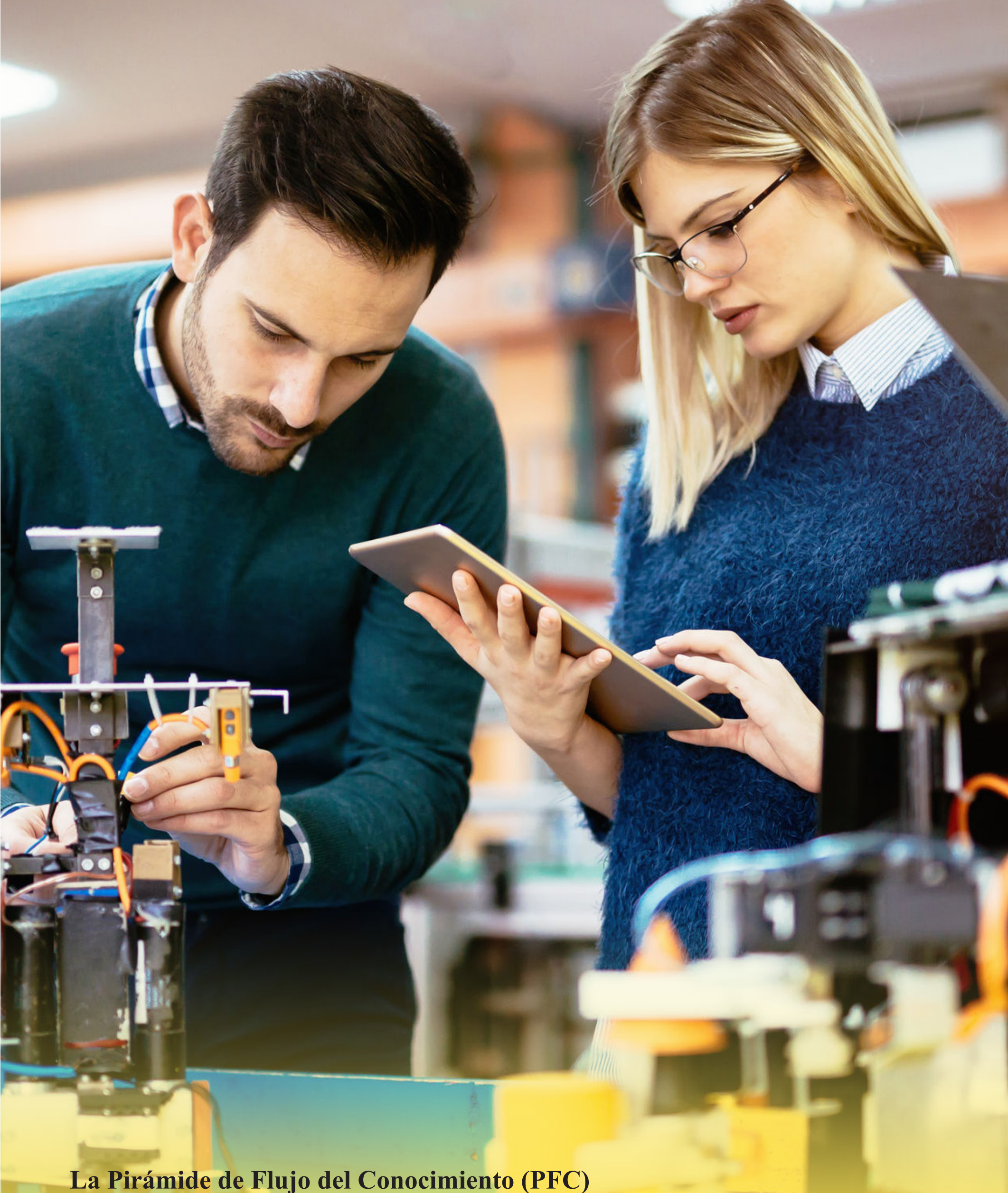

La Pirámide de Flujo del Conocimiento (PFC)

Una forma de integrar la transformación productiva y la educación técnica 


\title{
La Pirámide de Flujo del Conocimiento (PFC) Una forma de integrar la transformación productiva y la educación técnica
}

\author{
M.Sc. Ronald Bolaños Maroto \\ Escuela de Ingeniería Electromecánica \\ Universidad Fidélitas \\ San José, Costa Rica \\ rbolanos@ufidelitas. ac.cr
}

\begin{abstract}
An old Chinese proverb says: "What I hear, I forget; what I see, I remember; what I do, I learn". This wisdom continues to be valid in this 21 st century and even more so in this era characterized by the proliferation of new knowledge resulting from accelerated change in all areas of human activity. We are experiencing the Fourth Industrial Revolution, a process as amazing as it is challenging that inevitably leads to reinventing our society - the way we think, relate, and live, but also radically transform production systems, giving rise to a new paradigm that forces us to retrain our workforce at lightning speed, especially technicians and engineers.
\end{abstract}

This implies that we must manage to achieve more effective and faster ways to transfer knowledge. In MECSOFT we find one that we have been using and refining for 20 years. We call it the Pyramid of the Flow of Knowledge (PFC in Spanish); Its results have always been so encouraging that we believe it is very convenient and timely to share it with society, especially at this time when education is urgently reinvented.

MECSOFT is a company specialized in Computer Aided Design and Manufacturing and arises from a university research project with the idea of implementing this technology in the Costa Rican productive sector. The objective has been fulfilled thanks to the Pyramid of Knowledge Flow method.

Keywords: Cuarta Revolución Industrial, Educación Técnica, Transferencia de Tecnología, Transformación Productiva.

\section{Introducción}

La Cuarta Revolución Industrial avanza imparable cambiando todo a su paso; transforma la sociedad y redefine todas las formas de producción conocidas, lo que obliga a reentrenar sin demora a nuestra fuerza laboral, especialmente técnicos e ingenieros.

Esto implica que debemos generar nuevas y más efectivas formas de transferir el conocimiento y para contribuir con este empeño es que deseo compartir el modelo de transferencia de conocimiento que se utiliza en MECSOFT con mucho éxito.

MECSOFT es una empresa especializada en el Diseño y Manufactura Asistidos por Computador. Surge de un proyecto de investigación universitario con la idea de implementar esta tecnología en el sector productivo costarricense, algo que se logra con esta modalidad de transferencia del conocimiento que denomino: Pirámide de Flujo del Conocimiento.

Su efectividad hace prever que será la educación del futuro, aunque sus raíces se formaron desde la antigüedad, cuando las personas aprendían un oficio bajo la guía de un maestro.

Considero que su principal causa de éxito, descansa en el hecho de que al mismo tiempo en que se le transfiere el conocimiento al estudiante, se van desarrollando un conjunto de habilidades blandas, que son hoy en día cruciales para acceder, innovar y triunfar en los mercados cada vez más competitivos: pasión, curiosidad, el aprender a tomar riesgos calculados, no temer al fracaso y aprender de los errores.

Durante dos décadas, la "Pirámide de Flujo del Conocimiento" ha logrado que los técnicos "aprendan haciendo" guiados por expertos; eso ha dado enormes réditos a MECSOFT y a sus clientes, por eso ahora estoy convencido en la conveniencia de escalar este método a los centros de enseñanza técnica y a las carreras de ingeniería; porque creo que será un buen aporte, sobre todo en este momento en el que se está reinventando la educación.

\section{Metodología}

La metodología de PFC se desarrolla en cuatro etapas:

1. El Gurú de una empresa de tecnología que desarrollo una nueva forma de resolver un problema común en el sector productivo, explica al consultor como funciona la misma. 
2. El consultor determina si esta solución puede aplicarse con éxito en nuestro país. Si la respuesta es positiva, genera el material didáctico y recopila experiencias, para que con base en ellas el alumno aprenda como se usa esta nueva herramienta tecnológica.

3. El estudiante aprende y resuelve los problemas de la empresa interesada en aplicar la tecnología.

4. El estudiante enseña a los usuarios de la empresa como se usa la aplicación para hacer su trabajo.

Resumiendo, el consultor aprende del Gurú y traslada el conocimiento al estudiante y este a su vez lo traslada al usuario final, con lo que se logra culminar con éxito la transformación productiva de la empresa.

La relación entre consultor/profesor y aprendiz/estudiante ha tenido metodológicamente gran suceso. En lugar de tener un profesor, ahora el estudiante cuenta con la orientación de un consultor, una persona que sabe diagnosticar problemas en las empresas, sabe implementar nuevos conceptos tecnológicos para resolver esos problemas; además ya ha acumulado experiencia formando el personal en la implementación de esas nuevas tecnologías y sobre todo ama enseñar.

En mi larga trayectoria como profesor universitario, he podido comprobar que el estudiante le tiene más respeto a aquel profesor que además de la teoría, le proporciona su experiencia como consultor que ha tenido que sortear todo tipo de retos.

Después de revisar la bibliografía citada en las referencias, compruebo que si bien existen metodologías similares que ya se aplican en la educación técnica, no encuentro referencias al uso de la misma para lograr la transformación productiva de las empresas. Por eso no aparecen citas específicas que confirmen mis observaciones. Pero estoy convencido que esta metodología que se probó con éxito en las empresas para introducir nuevas herramientas informáticas en la solución de problemas de ingeniería, debe ser introducirla en las universidades en la formación de los ingenieros. Con ese fin y como director de la carrera de Ingeniería Electromecánica de la Universidad Fidélitas, estoy incorporando consultores como profesores en la carrera, como es el caso de Luis Chavarría y Emmanuel Coto. En el capítulo de Resultados comentaré sus experiencias como consultor/profesor en las aulas universitarias y la forma en que el método PFC coadyuva a lograr una mayor empleabilidad de los estudiantes.

\section{Desarrollo}

Desde el 2011 los más importantes medios de comunicación de Costa Rica, entre ellos, La Nación, El Financiero y Telenoticias, dieron a conocer como un grupo de supertécnicos de MECSOFT, lograron realizar ingeniería inversa con éxito para reparar un avión en COOPESA.
En aquel momento solo existían dos empresas en Estados Unidos capaces de realizar este proceso y para la BOEING fue una sorpresa que se pudiera realizar en Latinoamérica y particularmente en Costa Rica.

Justamente el supertécnico se diferencia del resto al tener un conocimiento profundo sobre un área específica de la tecnología, que le permite resolver problemas que un técnico normal no podría.

Como empresa durante años he compartido esta experiencia con las universidades públicas, animándolos a incorporar esta metodología en las carreras de ingeniería. Porque el negocio de MECSOFT no es educar y como empresario, a mí me gustaría con esta metodología se utilizará para desarrollar supertécnicos y superingenieros, que contribuyan a transformar productivamente a las empresas y al país.

Desde la primera década del 2000, MECSOFT se acercó al Colegio Técnico Cedes Don Bosco, en busca de un aliado estratégico, con el fin de incorporar tecnología de punta en sus capacitaciones. Esto ha tenido un gran suceso en esa institución, sin embargo, no se había logrado hacer lo mismo en ninguna institución universitaria. Esto me motivo a aceptar la dirección de Ingeniería Electromecánica en la Universidad Fidélitas, con el fin de encontrar la mejor forma de implementar esta metodología en la formación de ingenieros.

El método Pirámide de Flujo de Conocimiento, PFC, que funcionó en las empresas debería por tanto ser impartido en las universidades como una llave al futuro, un sello de garantía de que los jóvenes técnicos encontrarán un empleo bien remunerado y estable.

¿Qué es lo que más le preocupa a una universidad sobre todo privada?, que cuando los chicos se gradúen no consigan trabajo después de haber hecho una millonaria inversión económica y en tiempo.

Esta fórmula de formación técnica que hemos practicado en MECSOFT, PFC, hace que estos jóvenes estudiantes de áreas técnicas sean más buscados y valorados por el sector productivo. Démosle a esos jóvenes el conocimiento y herramientas que el sector productivo está ansioso de adquirir y ese será su mejor tarjeta de presentación.

El éxito de la PFC se basa en el "aprender haciendo". Un método que promueve la pasión, la curiosidad y el compromiso en el estudiante. Un viejo proverbio chino dice: "Lo que oigo, lo olvido; lo que veo, lo recuerdo; lo que hago, lo aprendo". Esta sabiduría sigue teniendo vigencia en este siglo XXI.

Cabe destacar que este tipo de aprendizaje no es un fin en sí mismo, sino un medio para el desarrollo integral de toda persona que aspire a ser un buen técnico en la Cuarta Revolución Industrial. 
Está claro que conforme surjan nuevas y más complejas tecnologías, el país va a requerir a los técnicos más calificados e innovadores, esos que "aprenden haciendo" a través de un proceso entretenido y retador, en el que siempre encuentran apoyo y orientación por parte de los expertos.

El otro factor de éxito de la Pirámide de Flujo de Conocimiento, PFC, como modelo de educación técnica, es que acelera la transformación productiva de las empresas (generalmente renuentes al cambio), a través de la oportuna articulación entre colegios técnicos, la universidad y las empresas. Su alineación logra hacer realidad aquel ideal de que las empresas cuenten con técnicos jóvenes ya formados que estén en condiciones de entender mejor la aplicación y gestión de esa avalancha de distintas tecnologías que llegan a los sectores productivos y a los mercados.

Este modelo permite formar ese técnico que rápidamente se convertirá en el "internal champion", capaz de demostrar que con el nuevo software se puede producir resultados 10 veces más rápido y le mostrará a los tomadores de decisiones, una efectividad hasta ahora desconocida por ellos. A la vez, genera un vigoroso efecto dominó ya que el resto de compañeros, al ver los resultados de ese "internal champion", querrán emularlo, conformando rápidamente una masa crítica que será el motor de la transformación productiva de la empresa y de paso mejora la educación técnica, algo impostergable en tiempos de la Cuarta Revolución Industrial.

Este nuevo escenario hace que de inmediato el empresario aspire a tener ya no un "internal champion" sino 10 , convencido de que eso elevará la productividad de su empresa en tiempos complejos y de altísima competitividad. Con la PFC gana el estudiante, gana la empresa y gana el país.

Ahí es donde yo veo que la PFC es a la vez y sobre todo un método para la rápida transformación productiva de las empresas, disparando su productividad pero también la competitividad del país. Y lo logra al revolucionar la educación técnica. Es la forma de tener gente mejor calificada que constituye el motor de cambio en las empresas.

La PFC tiene muchas similitudes con lo que se conoce como Educación Dual y también con lo que se denomina Formación Basada en Proyectos. Sin embargo, la gran diferencia estriba en que mientras que aquellos aprenden con "lo que ya se conoce", en la PFC se trata de "crear y de innovar con lo que viene"; valga aclarar que en todo el proceso el protagonista es el estudiante, porque asimila 5 veces más rápido el conocimiento que un profesional ya maduro que este trabajando en la empresa. Yo lo comparo al aprendizaje de un idioma, si se quiere tener personas que hablen perfectamente otra lengua, hay que enseñarles cuando aun son jóvenes. Lo mismo ocurre con las nuevas tecnologías de ingeniería, porque los ingenieros utilizan un lenguaje gráfico para comunicar sus ideas y la introducción de la informática, permite crear sistemas de información que usan modelos tridimensionales para representar las máquinas y edificios que se van a construir o mantener. Esto implica una forma diferente de hacer las cosas y resolver los problemas de ingeniería, que requiere entrenar los jóvenes desde la escuela, para lograr un dominio experto de estas nuevas herramientas.

La PFC se trata de un método que opera al revés, ya que impacta al proceso educativo pero desde la transformación productiva de las empresas, desde el punto de vista de las empresas y no de la universidad o del colegio vocacional.

Promueve la formación de técnicos que puedan tropicalizar los conceptos y herramientas a nuestra realidad, que sean técnicos pensantes, que desarrollen el pensamiento crítico necesario de resolver problemas bajo presión. Que tengan el conocimiento y que sepan utilizarlo.

\section{Resultados}

Para ilustrar el impacto que tiene la Pirámide de Flujo de Conocimiento en la educación técnica y en la transformación productiva de las empresas, he seleccionado el trabajo de dos consultores/profesores que aplican este método con sus estudiantes de las carreras de Ingeniería Electromecánica de la Universidad Fidélitas, considerada la universidad privada con mayor oferta en ingenierías en Costa Rica. Se trata de Luis Chavarría y Emmanuel Coto.

Para Chavarría, el principal diferenciador en el modelo de enseñanza profesor/consultor, es la orientación que se da a los estudiantes enfocada directamente en la resolución de problemas reales y con tecnología de punta. Se pasa de un modelo teórico en el cual solo se repite una clase magistral, a un modelo pragmático de involucramiento, empoderamiento y de co-creación. Aquí la obtención de una nota de calificación es el último de los resultados esperados.

Este consultor ha podido constatar de manera reiterada, que al enfrentarse los estudiantes a problemas reales y cercanos, escogidos por ellos mismos y validados por el profesor, los jóvenes se apoderan del problema, y quizás más importante aún, se motivan, y le dedican más tiempo a la clase de lo que normalmente harían. Incluso, muchas de las ideas son identificadas por los mismos estudiantes como proyectos candidatos a la formulación de modelos de negocio, dando pie a un verdadero esquema de aprendizaje e innovación que deriva más temprano que tarde en una cultura de emprendedurismo que sin duda dinamiza la economía de los países.

Entre los muchos casos de éxito que han cosechado los grupos de estudiantes de Chavarría, destacan los siguientes: un sistema de reanimación cardiopulmonar "RCP" automático que permite una aplicación de la maniobra controlada, continua y precisa hasta que se permita la estabilización del paciente. 
Por otro lado, el diseño de un exoesqueleto que permite al portador mejorar sus capacidades físicas para levantar más peso del soportado normalmente, así como cortar metal para rescatar a personas atrapadas; siendo este exoesqueleto monitoreado a través de la internet y permitiendo la visualización del estado de sus componentes con el uso de Realidad Aumentada.

En tercer lugar hay que destacar el desarrollo completo y funcional de los modelos 3D de una araña robot para su posterior programación de comportamiento en herramientas de desarrollo integrado a través de programación gráfica.

El éxito alcanzado por los estudiantes en estos y otros muchos proyectos, se debe en primer lugar al grado de motivación que despierta el método en ellos y que los lleva a dar más de lo esperado, investigando por su propia cuenta otras herramientas para resolver el problema que ellos mismos plantearon. Es importante aclarar que estos resultados se contrastan con los resultados de la metodología anteriormente aplicada en la misma clase, en la cual los estudiantes solo aprendían a dibujar planos en 2 dimensiones.

Por su parte, el consultor/profesor Emmanuel Coto, destaca que dentro de las técnicas utilizadas para la formación de ingeniería, especial atención se le da a la PFC, una metodología que empodera más al estudiante y ayuda a generar el instinto autodidacta y generación de conocimiento, es decir se trata de crear seres pensantes y con pensamiento crítico.

En un primer momento el consultor se enfrenta a una serie de situaciones comunes en la empresa, además dicha experiencia ayuda a identificar las debilidades de los recién egresados al llegar a la industria y a determinar cuáles habilidades requieren desarrollar, por ende, se pasa a generar una serie de experiencias que ayuden a que el estudiante refuerce los conceptos en los que han mostrado debilidades y que son los requeridos por los empleadores.

Por ejemplo el primer caso es en el curso de Diseño Asistido por Computador, donde se ha preparado una serie de materiales en formato PDF y video. En primera instancia el profesor es un guía que introduce conceptos y demuestra algunos usos pero posteriormente el estudiante tiene la oportunidad de avanzar a su ritmo siguiendo las practicas reflejadas en los archivos, lógicamente para aprobar y validar los conocimientos se pueden rendir algunas pruebas.

Otra etapa de la formación PFC, es acercar a los estudiantes a las empresas y otras instituciones que tienen laboratorios, mejorando así su percepción práctica y el reforzamiento de conceptos. Por ejemplo en el laboratorio de metalurgia los estudiantes tienen acercamientos y experiencias de ensayos de materiales con el INA, maquinado y deconformado de aceros con el ICE, TIASSA y Cedes Don Bosco, Fundición con DI Aceros y vidrio con Extralum.
Otra forma de desarrollar habilidades necesarias en las empresas es a través de proyectos sobre distintas situaciones que se asemejen a la realidad, por ejemplo en el curso de Mecánica del Sólido se les asigna cada cuatrimestre un proyecto en el que tienen que construir una maqueta en la que deben utilizar la materia de todo el curso; los estudiantes desarrollan una memoria de cálculo que justifica la fabricación como en una situación real y la maqueta se prueba con peso para comprobar su resistencia. Hasta el momento han desarrollado puentes, grúas pórtico, chasis de camión, ruedas de chicago y actualmente grúas de taller.

\section{Discusión de resultados}

Según los consultores Chavarría y Coto, los resultados con la metodología PFC han sido muchos y todos muy positivos. El entusiasmo en los grupos de estudiantes ha sido tan alto que en no pocas ocasiones en lugar de desarrollar el proyecto que se les pide, desarrollan dos y hasta tres.

El entusiasmo deriva de la dinámica propia del PFC que los va empoderando en su proceso de aprendizaje y les va aumentando la autonomía en la ejecución de sus proyectos, pero también en que la regla de oro es que se planteen problemas propios de su entorno o realidad cercana.

En el caso del RCP automático los muchachos habían investigado que a veces el proceso de resucitación se practica mal y la persona muere, o cuando lo hacen manual si lo hacen muy fuerte pueden quebrarle las costillas al paciente con el riesgo de perforar el pulmón $u$ otros órganos.

Así que se plantearon la resolución de un problema en el área de la salud que nos puede afectar a todos, incluyendo a familiares o a nosotros mismos. Eso los elevó el nivel de motivación y compromiso.

Usando esta metodología PFC y basados en el "Engineering Design Process", modelaron prototipos de cero y usaron algunas herramientas de programación. En el RCP automático se desarrolló el prototipo en $3 \mathrm{D}$, se colocó en un ambiente controlado y se verificó si funcionaría como se tenía planeado.

Estos RCP se colocan en las camillas de emergencia que se ubican en todas las organizaciones. El dispositivo es como una media luna que va de un extremo de la camilla al otro, con una tabletita que se le coloca encima de la persona y realiza el procedimiento de resucitación. Un diseño agradable y funcional que se puede aplicar in situ y monitorear en forma remota.

La tableta determina si el paciente es masculino o femenino, y brinda indicadores como rango de edad, estatura, peso, y calcula el movimiento y cantidad de fuerza que requiere para resucitar al paciente. 
El hecho de que los estudiantes se planteen un problema sobre una situación cercana es clave. Uno de ellos tiene un recién nacido y no sabían qué hacer con los pañales que ocupan volumen y contaminan, pues ahora están trabajando en una máquina para descomponer los materiales de los pañales y darles otro uso.

El conocimiento, las habilidades y valores que adquieren en el proceso ayuda a los muchachos a conseguir trabajo y a destacar en ellos como el principal motor en la transformación productiva de las empresas.

Soy un convencido que para insertarnos con éxito en la Cuarta Revolución Industrial la tarea más urgente es la reinvención de la educación técnica. La forma de hacerlo es lograr que el protagónico no lo tenga el educador sino al estudiante. ¿Cómo?, bueno, si los consultores comienzan a meterse en el proceso educativo entendiendo cuál es la mejor forma de motivar a los estudiantes, ellos pueden comenzar a general material que podemos luego subir a la nube, y el maestro no será el que más sepa sino un facilitador. El consultor tiene poco tiempo pero si pone su experiencia en material didáctico y se lo facilita al estudiante, tenemos que generar el conocimiento y las rutas necesarias para que el estudiante aprenda por sí mismo.

\section{Referencias}

1. Wagner Tony., (2014), Creando Innovadores, La formación de los jóvenes que cambiarán el mundo. Editor Kolima, versión Kindle. USA.

2. AOUN, JOSEPH E., (2017), Robot-Proof: Higher Education in the age of Artificial Intelligence. The MIT Press. USA.
3. Naranjo Claudio, (2013), Cambiar la Educación para cambiar al mundo. Editorial La Llave, Chile.

4. Lizette Brenes Bonilla,(2019), Crear una vida extraordinaria ante la Cuarta Revolución Industrial. Fundepredi, UNED. Costa Rica.

5. Benavides, Carlos A, (1998), Tecnología, innovación y empresa. Madrid, España, Pirámide.

6. Ruiz, Ángel, (2001), El destino de Costa Rica y la educación superior. El escenario histórico del país, la educación y el papel de la universidad. Editorial Universidad de Costa Rica. Costa Rica.

7. Canito, J.L. Romero, A. (2016) Inventor 2017 (Manual Imprescindible). Madrid, España. Anaya Multimedia.

8. Popov, Egor P. (2006). "Introducción a la Mecánica de Sólidos”. Editorial Limusa, México.

9. Timoshenko, S. y Young, D. H. "Engineering Mechanics". Mc Graw- Hill, New York.

10. Budymas R., Nisbeth K., (2015). Diseño en Ingeniería Mecánica, Décima edición, México: Mc Graw Hill.

11. Hamrock B. (2016). Elementos de Máquinas, Primera edición. México: Mc Graw Hill.

12. Norton R.L. (2016). Diseño de Máquinas, Cuarta edición. México: Pearson.

13. NASA STEM ENGAGEMENTS. https://www.nasa.gov/ audience/foreducators/bes t/edp.html.

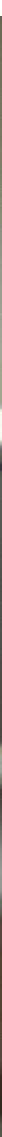

